\title{
The CERN Neutrino Platform
}

\author{
Stefania Bordoni* \\ CERN \\ E-mail: stefania.bordoniecern.ch
}

The long-baseline neutrino programme has been classified as one of the four highest-priority scientific objectives in 2013 by the European Strategy for Particle Physics. The Neutrino Platform is the CERN venture to foster and support the next generation of accelerator-based neutrino oscillation experiments.

Part of the present CERN Medium-Term Plan, the Neutrino Platform provide facilities to develop and prototype the next generation of neutrino detectors and contribute to unify the European neutrino community towards the US and Japanese projects. A significative effort is made on R\&D for LAr TPC technologies: two big LAr TPC prototypes for the DUNE far detector are under construction at CERN. Those detectors will be exposed in 2018 to an entirely new and NP-dedicated beam-line from the SPS which will provide electron, muon and hadron beams with energies in the range of sub-GeV to a few $\mathrm{GeV}$. Other projects are also presently under development: one can cite the refurbishing and shipping to the US of the ICARUS detector and the construction of Baby MIND, a magnetised muon spectrometer to be located on the T2K beam line.

In this talk the status of the CERN Neutrino Platform activities will be presented as well as an overview of the future projects currently under discussion.

The European Physical Society Conference on High Energy Physics

5-12 July, 2017

Venice

${ }^{*}$ Speaker. 


\section{Introduction}

In 2013, following the recommendation of the European Strategy of Particle Physics [1], CERN started a programme to support the activities toward the next generation of short and long base-line neutrino oscillation experiments: the Neutrino Platform (NP). Part of the CERN Medium Term plan, the NP is contributing to both Japanese and American projects by providing crucial know-how, infrastructures and facilities, together with the fostering of the European contribution on the concerned experiments.

\section{Current activities}

The current NP activities are organised in five projects which are related either to the experiments in Japan as T2K, T2K-II [2] and HyperK [3] or to the activities in the US towards the short baseline Neutrino program at Fermilab (SBN) [4], the Long Baseline Neutrino Facility (LBNF) and the Deep Underground Neutrino Experiment (DUNE) [5].

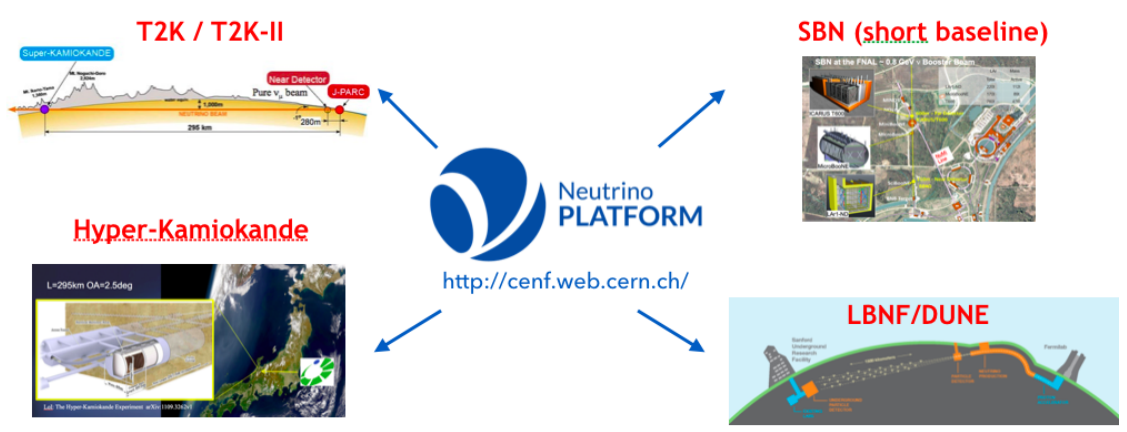

Figure 1: The CERN NP and its involvement on the current and future LBN experiments.

\subsection{The NP and Japan}

The NP has participated to the R\&D, the design and the finalisation of the Baby MIND detector, a magnetised muon spectrometer which will be located on the T2K beam-line downstream of the WAGASCI modules at the J-PARC T59 experiment experiment [6] (Figure 2a).

The goal of Baby MIND is to precisely identify the charge of the muons issued from the charged current neutrino interactions in the WAGASCI Carbon or Oxygen target. The spectrometer is composed by 33 magnet modules interleaved with 18 scintillator modules. The design of the detector and its final layout, that is how to distribute magnet and scintillator modules, have been optimised to maximise the lever arm necessary to correctly identify the charge of the muon even in case of multiple scattering down to $200 \mathrm{MeV} / \mathrm{c}$. The NP has contributed to the design of the magnet modules characterised by a two-slit design (Figure 2b)[7]. Each module is magnetised individually using normal conducting coils wound on the surface of each plate and providing a very uniform $\mathrm{B}$ field in the central zone of the plate.

The detector was completed at the start of Summer 2017 and then exposed to charged particle beams at the T9 CERN facilities (Figure 2c). The shipment to Japan is foreseen for beginning of October 2017 while the first data taking is expected for Spring 2018. 


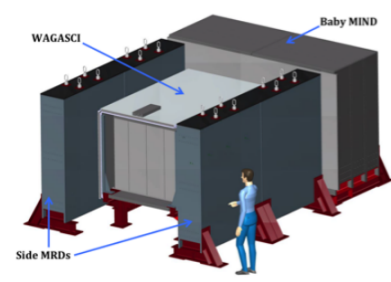

(a)

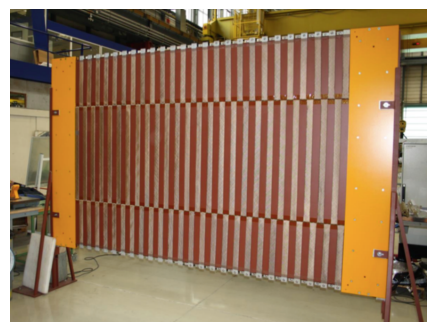

(b)

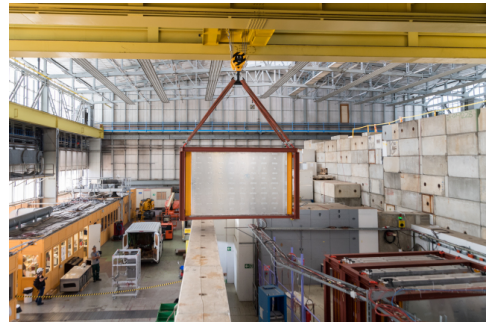

(c)

Figure 2: 2a The layout of the WAGASCI experiment; 2b A Baby MIND magnet module fully assembled; 2c Baby MIND being installed in the CERN T9 test beam area.

\subsection{The NP and the US}

\section{The ICARUS detector and the SBN program at Fermilab}

The NP is deeply involved in the activities to refurbish, overhaul and ship the ICARUS detector to Fermilab. The ICARUS detector [8], a 800 tons Liquid Argon (LAr) Time Projection Chamber (TPC), after a successful operation between 2009 and 2013, will be the far detector for the SBN program [4] at Fermilab. Several activities finalised to adopt new technologies more suitable to surface operations (as at Fermilab) has been performed: the construction of two new cold vessels made of extruded aluminium, the refurbishing of the cryogenics and purification system, the upgrade of the light collection system to ensure better space/time event localisation capabilities, the integration of new faster and high performance readout electronics and finally the design of an external Cosmic Ray Tagging System.

All activities have been carried out at CERN in collaboration with INFN. The refurbishing activities have been completed in by the end of Winter 2017 and the detector shipped to Fermilab in July ready to be placed in the pit.

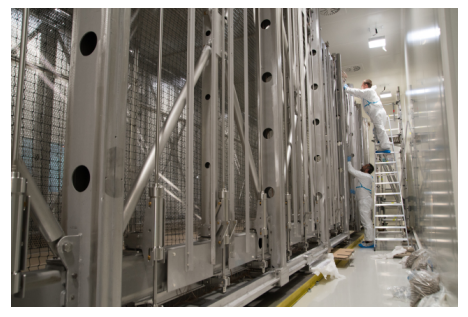

(a)

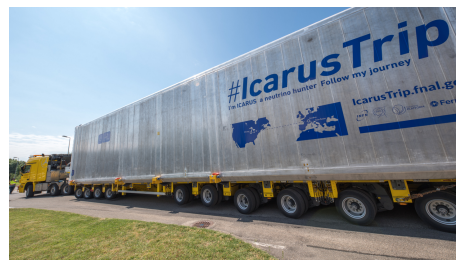

(b)

Figure 3: 3a One of the two TPC ICARUS modules during its refurbishment at CERN; $3 \mathrm{~b}$ One of the two ICARUS module (cold vessel and detector) on the track during the shipment to the US.

\section{The protoDUNE detectors and LBNF/DUNE}

The NP is deeply involved in the preparation of both LBNF and DUNE. Two gigantic prototypes for the far detector of DUNE are being constructed at CERN. Those detectors of about $8 \times 8 \times 8 \mathrm{~m}^{3}$ each, called protoDUNE, are liquid argon TPC and aim to test two different technologies $[9,10]$. Several R\&D activities have been performed by the NP with strong synergies be- 
tween the two projects, as for example the design of the extruded aluminium profiles for the field cage structure, the design of high-voltage feed throughs able to support very high voltages (up to $-300 \mathrm{kV}$ ), the design and test of the resistive cathode concept. Furthermore, a new experimental area $\left(\sim 53000 \mathrm{~m}^{3}\right)$ has been built to host the protoDUNE detectors and test them with tagged beams. For this purpose two new tertiary beam lines from the SPS have been designed to deliver very-low energy particle beams ( 1 to $6 \mathrm{GeV}$ ), energies of interest for the DUNE experiment (Figure 4a).

In support to both DUNE (protoDUNE and future far detector modules) and as well the SBL activities, the NP has created a strong LAr cryogenics group working in close collaboration with Fermilab, allowing for extreme purity of the liquid $(<100 \mathrm{ppt})$, sophisticated safety systems and new purge valves. A new technology, based on the GTT membrane brevet [11] usually employed for transport and storage of Liquified Natural Gas (Figure 4b), has been experimented for the first time for large volume cryostats in particle physics. Both the cryogenic system and the new cryostat technology have been already successfully tested in the $3 \times 1 \times 1 \mathrm{~m}^{3}$ dual phase demonstrator. The NP has obtained the responsibility for the design and construction of the cryostats and cryogenic system for the DUNE far detector, and for the detectors participating to the SBN program (SBND and ICARUS). Other facilities like clean rooms, assembly areas and R\&D laboratories are also provided by the NP to support the protoDUNE activities. Figure $4 \mathrm{c}$ shows one of the warm cryostats of a protoDUNE detector during its construction at the new experimental area.

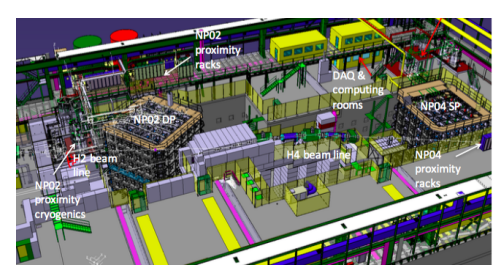

(a)

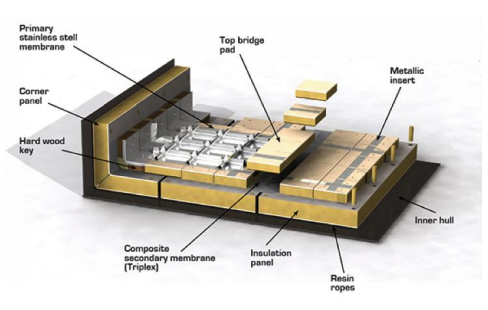

(b)

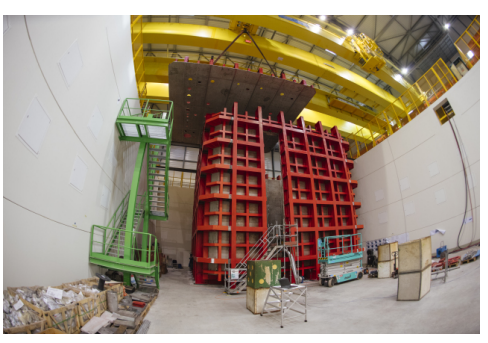

(c)

Figure 4: 4a The new NP experimental area; $4 \mathrm{~b}$ cross-section of the GTT membrane technology; $\mathrm{f}$ $4 \mathrm{c}$ one of the protoDUNE cryostats during its construction.

\section{Other projects and conclusion}

The NP is also involved in activities intended to support the data taking of the protoDUNE detectors and the treatment of large amount of data $(\sim 1 \mathrm{~Tb} / \mathrm{s})$, fostering the connection with the LHC expertise. For example, a new data acquisition system based on Front End Link EXchange cards (FELIX) [12] developed by the ATLAS collaboration is being integrated and tested in protoDUNE, as well the data storage and the processing operations are tightly connected to the know-how acquired with the LHC experiments. Software-based activities to improve the simulation of the incoming beams, the reconstruction of events in liquid argon and the preparation of data analyses are also performed by the NP in collaboration with the CERN EP-NU group.

Looking forward, several projects are currently in the pipe-line to become part of the NP as for example ArgonCube [13], ENUBET [14], the participation to the upgrade of the off-axis 
near detector of T2K and R\&D activities for a high-pressure TPC intended to perform neutrino nucleus cross-section measurements with different targets [15]. In parallel, a new initiative intended to participate to the study and the design of the near detectors for the next generation of LBN experiment has been recently started [16] by the NP.

To conclude, the NP since 2015 has contributed to foster the activities towards acceleratorbased neutrino experiments, strengthening the European contribution but also developing synergies among different neutrino projects and with the LHC experiments.

\section{References}

[1] The European Strategy of Particle Physics 2013, Update 2013, Phys. Scr. 2013014019.

[2] The T2K collaboration, Proposal for an Extended Run of T2K to $20 \times 10^{21}$ POT, arXiv:1609.04111.

[3] The Hyper-Kamiokande working group , A Long Baseline Neutrino Oscillation Experiment Using J-PARC Neutrino Beam and Hyper-Kamiokande, arXiv:1412.4673.

[4] R. Acciarri et al., A Proposal for a Three Detector Short-Baseline Neutrino Oscillation Program in the Fermilab Booster Neutrino Beam, arXiv:1503.01520.

[5] The DUNE collaboration, Long-Baseline Neutrino Facility (LBNF) and Deep Underground Neutrino Experiment (DUNE) Conceptual Design Report Volume 2: The Physics Program for DUNE at LBNF, arXiv:1512.06148.

[6] E. Noah et al., The WAGASCI experiment at J-PARC to measure neutrino cross-sections on water, PoS (EPS-HEP2015) 292, 2015.

[7] G. Rolando et al., New and Optimized Magnetization Scheme for the Baby Magnetized Iron Neutrino Detector at J-PARC, IEEE Transactions on Magnetics, Vol. 53, No.5, May 2017.

[8] Antonello, M., et al. Experimental search for the LSND anomaly with the ICARUS detector in the CNGS neutrino beam Eur. Phys. J. C (2013) 73: 2345.

[9] The DUNE Collaboration, The Single-Phase ProtoDUNE Technical Design Report, arXiV: 1706.07081.

[10] L. Agostino et al., LBNO-DEMO: Large-scale neutrino detector demonstrators for phased performance assessment in view of a long-baseline oscillation experiment, arXiv:1409.4405.

[11] GTT Membrane Technologies, http://www.gtt-training.co.uk/gtt-membrane-technologies

[12] The ATLAS Collaboration, FELIX: the detector readout upgrade of the ATLAS experiment, ATL-DAQ-PROC-2017-008.

[13] C. Amsler et al., LoI ArgonCUBE: a novel, fully-modular approach for the realisation of large mass liquid argon TPC neutrino detectors, CERN-SPSC-2015-009.

[14] A. Berra et al., EoI Enabling precise measurements of flux in accelerator neutrino beams: the ENUBET project, CERN-SPSC-2016-014.

[15] P. Hamacher-Baumann et al., EoI Near detectors based on gas TPCs for neutrino long baseline experiments, CERN-SPSC-2017-002.

[16] https://twiki.cern.ch/twiki/bin/view/CENF/NearDetector 